Article

\title{
Reduction Operators and Exact Solutions of Variable Coefficient Nonlinear Wave Equations with Power Nonlinearities
}

\author{
Dingjiang Huang *, Yan Zhu and Qinmin Yang \\ Department of Mathematics, East China University of Science and Technology, Shanghai 200237, China; \\ zy0205s@163.com (Y.Z.); qmyang@ecust.edu.cn(Q.Y.) \\ * Correspondence: djhuang@ecust.edu.cn; Tel.: +86-21-6425-3147 \\ Academic Editor: Roman M. Cherniha \\ Received: 13 October 2016; Accepted: 14 December 2016; Published: 22 December 2016
}

\begin{abstract}
Reduction operators, i.e., the operators of nonclassical (or conditional) symmetry of a class of variable coefficient nonlinear wave equations with power nonlinearities, are investigated within the framework of a singular reduction operator. A classification of regular reduction operators is performed with respect to generalized extended equivalence groups. Exact solutions of some nonlinear wave models, which are invariant under certain reduction operators, are also constructed.
\end{abstract}

Keywords: symmetry analysis; reduction operators; equivalence group; nonlinear wave equation; exact solutions

\section{Introduction}

The investigation of geometric and algebraic structures, as well as exact solutions of nonlinear wave equations plays an important role in the study of nonlinear physical phenomena. Generally, when the equation under consideration is an exact solvable or integrable model (e.g., KdVequation), we can use many efficient methods, such as inverse scattering transform, Bäcklund and Darboux transform, Painleve analysis, nonlocal symmetry reductions, and so on (see, for example, References [1-5] and the references cited therein), to construct its exact solutions. While, if the equation is a non-integrable model, we usually turn to use the so-called Lie group analysis and its extension $[6,7]$ to investigate it.

In this paper, we study reduction operators, i.e., the operators of nonclassical (or conditional) symmetry associated with a class of non-integrable variable coefficient nonlinear wave equations with power nonlinearities of the form:

$$
f(x) u_{t t}=\left(g(x) u^{n} u_{x}\right)_{x}+h(x) u^{m},
$$

where $f=f(x), g=g(x)$ and $h=h(x)$ are three arbitrary functions, $f g \neq 0, n$ and $m$ are arbitrary constants, $t$ is the time coordinate and $x$ is the one-space coordinate. The linear case is excluded from consideration because it was well investigated. We also assume the variable wave speed coefficient $u^{n}$ to be nonlinear, i.e., $n \neq 0$. The case $n=0$ is quite singular and will be investigated separately.

Many specific nonlinear wave models derived from mechanics and engineering, such as the one-dimensional gas flow, longitudinal wave propagation on a moving thread line and electromagnetic transmission line, and so on, can be reduced to Equation (1) (see [8] pp. 50-52 and [9]). Since the 1970s, Lie symmetries and invariant solutions of various kinds of quasi-linear wave equations in two independent variables that intersect Class (1) have been investigated $[7,10-24]$ because of the importance of the wave equation for various applications. Recently, we have presented a complete Lie symmetry and conservation law classification of Class (1) $[25,26]$. Classical Lie symmetry reduction 
and invariant solutions of some variable coefficients wave models, which are singled out from the classification results, are also investigated [25].

In general, using classical Lie symmetries' reduction of partial differential equations can provide part of the exact solutions of these equations $[6,27,28]$. In order to find more other types of exact solutions, one should generalize Lie's original reduction. The first approach to such a generalization was present by Bluman and Cole in 1969 [29] (see also [7]) in which they introduced a wider class of infinitesimal generators than Lie symmetries. Later, such infinitesimal generators, were named nonclassical symmetries [8,30-33] or Q-conditional symmetries [34-41] and were also extended by many authors to some concepts, such as conditional symmetries [34] (the notion 'conditional symmetry' used in this well-known monograph does not coincide with the notion 'non-classical', but is an essential generalization of non-classical symmetry (see Chapter 5.7 therein)), weak symmetry [33], differential constraints, etc. [42-44]. Recently, Boyko, Kunzinger and Popovych [45,46] named it as 'reduction operators' and present a novel framework, namely singular reduction operators or singular reduction modules, for finding an optimal way of obtaining the determining equation of conditional symmetries. As an application, they have investigated the properties of singular reduction operators for a number of $(1+1)$-dimensional evolution equations and a specific wave equations by using this new framework [46]. However, for more general nonlinear wave Equation (1), there exist no general results. In this paper, we employ Popovych's singular reduction operators theory to investigate the properties of nonclassical symmetries of Class (1). We propose a complete classification of regular reduction operators for (1) with respect to generalized extended equivalence groups and construct some non-Lie exact solutions for the nonlinear wave model, which are invariant under certain reduction operators. It should be noted that the terminology 'non-classical symmetry' and 'Q-conditional symmetry' is still used very widely in the literature in contrast to 'reduction operators'; however, we prefer to use this relatively new terminology.

The rest of paper is organized as follows. In Section 2, singular reduction operators and, in particular, regular reduction operators classification for the class under consideration are investigated. Section 3 contains the nonclassical symmetry reduction of some nonlinear wave models. New non-Lie exact solutions of the models are constructed by means of the reduction. Conclusions and a discussion are given in Section 4 .

\section{Nonclassical Symmetries}

Nonclassical symmetries of Class (1) are performed in the framework of the singular reduction operator [46]. All necessary objects (singular and regular reduction operator, etc.) can be found there [46]. Before we proceed with the investigation, we can first simplify the class (1). Using the transformation:

$$
\tilde{t}=t, \quad \tilde{x}=\int \frac{d x}{g(x)}, \quad \tilde{u}=u
$$

from Theorem 1 in [25], we can reduce Equation (1) to $\tilde{f}(\tilde{x}) \tilde{u}_{\tilde{t} \tilde{t}}=\left(\tilde{u}^{n} \tilde{u}_{\tilde{x}} \tilde{x}+\tilde{h}(\tilde{x}) \tilde{u}^{m}\right.$, where $\tilde{f}(\tilde{x})=g(x) f(x), \tilde{g}(\tilde{x})=1$ and $\tilde{h}(\tilde{x})=g(x) h(x)$. Thus, without loss of generality, we can restrict ourselves to investigation of the equation:

$$
f(x) u_{t t}=\left(u^{n} u_{x}\right)_{x}+h(x) u^{m} .
$$

For convenience, we can further rewrite it as the form:

$$
L[u]:=f(x) u_{t t}-\left(u^{n} u_{x}\right)_{x}-h(x) u^{m}=0 .
$$

All results on symmetries and solutions of Class (3) or (4) can be extended to Class (1) with transformations (2).

According to the algorithm in [29], we seek a reduction operator of Class (4) in the form: 


$$
Q=\tau(t, x, u) \partial_{t}+\xi(t, x, u) \partial_{x}+\eta(t, x, u) \partial_{u}, \quad(\tau, \xi) \neq(0,0),
$$

which is a first-order differential operator on the space $\mathbb{R}^{2} \times \mathbb{R}^{1}$ with coordinates $t, x$ and $u$, where the coefficients $\tau$ and $\xi$ do not simultaneously vanish. This operator allows one to construct an ansatz reducing the original Equation (4) to an ordinary differential equation. The conditional invariance criterion [32,36,41] for Equation (4) to be invariant with respect to the operator (5) reads as:

$$
\left.\operatorname{pr}^{(2)} Q(L[u])\right|_{{\mathcal{L} \cap \mathcal{Q}_{(2)}}=0}
$$

where $\mathrm{pr}^{(2)} Q$ is the usual second-order prolongation [6,28] of the operator $(5), \mathcal{L}$ is the manifold in the second-order jet space $J^{(2)}$ determined by the wave equation $L[u]=0$ and $\mathcal{Q}_{(2)} \subset J^{(2)}$ is the the first prolongation of the invariant surface condition:

$$
Q[u]:=\tau u_{t}+\xi u_{x}-\eta=0 .
$$

The system $\mathcal{Q}_{(2)}$ consists of (7) and the equations obtained by $t$ - and $x$-differentiation of (7).

Below, according to the singular reduction operator theory [46], we first partition the set of reduction operators of Class (4) into two subsets, i.e., the singular reduction operator and the regular one. Then, we utilize the two kinds of operators to derive determining equations (overdetermined system of nonlinear PDEs with respect to the coefficients of the reduction operator (5)) from the conditional invariance criterion (6) separately. Solving the two systems, we can obtain the final reduction operators. In particular, we will present an exhausted classification of the regular operators of class (4) by solving the corresponding determining equations. In general, every Lie symmetry operator is also a reduction operator. Therefore, in this paper, we will concentrate on the regular reduction operators, which are inequivalent to Lie symmetry operators, called nontrivial.

\subsection{Singular Reduction Operators}

Using the procedure given by [46], we can obtain the following proposition.

Proposition 1. A necessary and sufficient condition for a vector field $Q=\tau(t, x, u) \partial_{t}+\xi(t, x, u) \partial_{x}+$ $\eta(t, x, u) \partial_{u}$, which is singular for the differential function $L=f(x) u_{t t}-\left(u^{n} u_{x}\right)_{x}-h(x) u^{m}$ from Equation (4), is that $\tilde{\xi}^{2} f(x)=\tau^{2} u^{n}$.

Proof. Suppose that $\tau \neq 0$, and using the characteristic equation $\tau u_{t}+\xi u_{x}-\eta=0$, we can obtain:

$$
\begin{gathered}
u_{t}=\frac{\eta}{\tau}-\frac{\xi}{\tau} u_{x} \\
u_{t t}=\left(\frac{\eta}{\tau}\right)_{t}-\left(\frac{\xi}{\tau}\right)_{t} u_{x}+\left[\left(\frac{\eta}{\tau}\right)_{u}-\left(\frac{\xi}{\tau}\right)_{u} u_{x}\right]\left(\frac{\eta}{\tau}-\frac{\xi}{\tau} u_{x}\right)-\left(\frac{\xi}{\tau}\right)\left[\left(\frac{\eta}{\tau}\right)_{x}+\left(\frac{\eta}{\tau}\right)_{u} u_{x}-\left(\frac{\xi}{\tau}\right)_{x} u_{x}\right. \\
\left.-\left(\frac{\xi}{\tau}\right)_{u} u_{x}^{2}-\left(\frac{\xi}{\tau}\right) u_{x x}\right] .
\end{gathered}
$$

Now, we substitute the second equation of the above system into $L$; we can get a differential function:

$$
\begin{aligned}
\tilde{L}=[ & \left.f(x)\left(\frac{\xi}{\tau}\right)^{2}-u^{n}\right] u_{x x}+f(x)\left\{\left(\frac{\eta}{\tau}\right)_{t}-\left(\frac{\xi}{\tau}\right)_{t} u_{x}+\left[\left(\frac{\eta}{\tau}\right)_{u}-\left(\frac{\xi}{\tau}\right)_{u} u_{x}\right]\left(\frac{\eta}{\tau}-\frac{\xi}{\tau} u_{x}\right)\right. \\
& \left.-\left(\frac{\xi}{\tau}\right)\left[\left(\frac{\eta}{\tau}\right)_{x}+\left(\frac{\eta}{\tau}\right)_{u} u_{x}-\left(\frac{\xi}{\tau}\right)_{x} u_{x}-\left(\frac{\xi}{\tau}\right)_{u} u_{x}^{2}\right]\right\}-n u^{n-1} u_{x}^{2}-h u^{m} u_{x} .
\end{aligned}
$$

With the aid of Definition 4 of the singular vector field in [46], we derive that a necessary and sufficient condition for ord $\tilde{L}<2$ is that $f(x)\left(\frac{\tilde{\xi}}{\tau}\right)^{2}-u^{n}=0$. 
Therefore, for any $f, h, n$ and $m$ with $f u^{n}>0$, there exist exactly two sets of singular vector fields in the reduced form for the differential function $L=f(x) u_{t t}-\left(u^{n} u_{x}\right)_{x}-h(x) u^{m}$ from Equation (4), i.e., $S=\left\{\partial_{t}+\sqrt{u^{n} / f} \partial_{x}+\hat{\eta} \partial_{u}\right\}$ and $S^{*}=\left\{\partial_{t}-\sqrt{u^{n} / f} \partial_{x}+\hat{\eta} \partial_{u}\right\}$, where $\hat{\eta}=\frac{\eta}{\tau}$. Any singular vector field of $L$ is equivalent to one of the above fields. Because the singular sets are mapped to each other by alternating the sign of $x$, we can just choose one of them for consideration.

Proposition 2. For any variable coefficient nonlinear wave equations in the form of (4), there exists exactly one set of singular vector fields in the reduced form, i.e., $S=\left\{\partial_{t}+\sqrt{u^{n} / f} \partial_{x}+\hat{\eta} \partial_{u}\right\}$ for the differential function $L=f(x) u_{t t}-\left(u^{n} u_{x}\right)_{x}-h(x) u^{m}$ from Equation (4).

Thus, for an equation from Class (4) and the operator $\partial_{t}+\sqrt{u^{n} / f} \partial_{x}+\eta \partial_{u}$, considering its conditional invariance criterion leads to:

Theorem 1. Every singular reduction operator of an equation from Class (4) is equivalent to an operator of the form:

$$
Q=\partial_{t}+\sqrt{u^{n} / f(x)} \partial_{x}+\eta(t, x, u) \partial_{u}
$$

where the real-valued function $\eta(t, x, u)$ satisfies the system of determining equations:

$$
\begin{aligned}
& \left(-1 / 2 n h u^{m-1}-2 f \eta_{t u}-2 f \eta \eta_{u u}\right) \sqrt{u^{n} / f}+\left(3 / 4 f_{x}^{2} / f-1 / 2 f_{x x}\right)\left(u^{n} / f\right)^{3 / 2} \\
& +\left(-1 / 4 n^{2} \eta^{2} u^{n-2}-n \eta \eta_{u} u^{n-1}+1 / 2 n \eta^{2} u^{n-2}\right)\left(u^{n} / f\right)^{-1 / 2}-n \eta_{x} u^{n-1} \\
& -1 / 2 n \eta u^{n-1} f_{x} / f-2 \eta_{x u} u^{n}=0 \\
& -\eta_{x x} u^{n}-n \eta \eta_{x} u^{n-1}\left(u^{n} / f\right)^{-1 / 2}+h \eta_{u} u^{m}+2 f \eta \eta_{t u}+f \eta^{2} \eta_{u u} \\
& -m h \eta u^{m-1}+f \eta_{t t}+\left(h f_{x} / f-h_{x}\right) u^{m} \sqrt{u^{n} / f}=0 .
\end{aligned}
$$

Remark 1. Equation (8) is highly nonlinear, and it is difficult to solve; hence, we do not discuss it here and will try to investigate it in a future publication.

\subsection{Regular Reduction Operators}

The above results for singular reduction operators of the class of nonlinear wave Equation (4) show that the regular case of the natural partition of the corresponding sets of reduction operators for the equation under consideration is singled out by the conditions $\xi \neq \pm \sqrt{u^{n} / f} \tau$. We factorize it with respect to the equivalence relation of vector fields, then we can derive the defining conditions of the regular subset of reduction operator, that is $\tau=1, \xi \neq \pm \sqrt{u^{n} / f}$. Hence, we have:

Proposition 3. For any variable coefficient nonlinear wave equations in the form of (4), there exists exactly one set of regular vector fields in the reduced form, i.e., $S=\left\{\partial_{t}+\hat{\xi} \partial_{x}+\hat{\eta} \partial_{u}\right\}$ with $\hat{\xi} \neq \pm \sqrt{u^{n} / f}$ for the differential function $L=f(x) u_{t t}-\left(u^{n} u_{x}\right)_{x}-h(x) u^{m}$ from Equation (4).

Taking into accountant the conditional invariance criterion for an equation from Class (4) and the operator $\partial_{t}+\xi(t, x, u) \partial_{x}+\eta(t, x, u) \partial_{u}$ with $\xi(t, x, u) \neq \pm \sqrt{u^{n} / f}$, we can obtain the following determining equations for the coefficients $\xi$ and $\eta$ :

$$
\begin{aligned}
& \xi_{u}=0, \quad 2 f \xi_{t}-n \eta u^{n-1}+\left(2 \xi_{x}+\xi f_{x} / f\right) u^{n}=0, \\
& \left(2 n \xi_{x}-n \eta_{u}+n \xi f_{x} / f\right) u^{n-1}+\left(n \eta-n^{2} \eta\right) u^{n-2}-\eta_{u u} u^{n}+f \xi^{2} \eta_{u u}=0, \\
& 2 f \xi_{t} \xi_{x}-2 f \xi_{t} \eta_{u}-2 n \eta_{x} u^{n-1}-f \xi_{t t}-2 f \tilde{\xi} \eta \eta_{u u}-2 f \xi_{t u}+\left(\xi_{x x}-2 \eta_{x u}\right) u^{n}=0, \\
& \left(\xi h f_{x} / f-\xi h_{x}+h \eta_{u}\right) u^{m}+f \eta^{2} \eta_{u u}+2 f \eta \eta_{t u}-2 f \xi_{t} \eta_{x}-\eta_{x x} u^{n}+f \eta_{t t}-m h \eta u^{m-1}=0 .
\end{aligned}
$$

From the first two equations of System (9), we have:

$$
\xi=\xi(t, x), \quad \eta=\frac{2}{n} f \xi_{t} u^{1-n}+\frac{1}{n}\left(2 \xi_{x}+\xi \frac{f_{x}}{f}\right) u
$$


Substituting the above formulas into the last three equations of System (9), we have the following assertion.

Theorem 2. Every regular reduction operator of an equation from Class (4) is equivalent to an operator of the form:

$$
Q=\partial_{t}+\xi(t, x) \partial_{x}+\eta(t, x, u) \partial_{u} \quad \text { with } \quad \eta(t, x, u)=\frac{2}{n} f \xi_{t} u^{1-n}+\frac{1}{n}\left(2 \xi_{x}+\xi \frac{f_{x}}{f}\right) u
$$

where the real-valued function $\xi(t, x)$ satisfies the overdetermined system of partial differential equations:

$$
\begin{aligned}
& 2(1-n) f \xi_{t}=0, \quad 2(1-n) f^{2} \xi^{2} \xi_{t}=0, \quad 8(1-n) f^{3} \xi^{2}=0, \\
& 4(1-n)\left[\left(f \xi_{t}\right)^{2}-f^{2} \xi \xi_{t}\left(2 \xi_{x}+\xi \frac{f_{x}}{f}\right)+f^{2} \xi \xi_{t t}\right]=0 \\
& \xi_{x x}-2\left(1+\frac{1}{n}\right)\left(2 \xi_{x}+\xi \frac{f_{x}}{f}\right)_{x}=0 \\
& 2 f \xi_{t} \xi_{x}-\frac{2}{n} f \xi_{t}\left(2 \xi_{x}+\xi \frac{f_{x}}{f}\right)-4\left(f \xi_{t}\right)_{x}-f \xi_{t t}-\frac{2}{n} f \xi\left(2 \xi_{x}+\xi \frac{f_{x}}{f}\right)_{t}-\frac{4}{n}(1-n)\left(f \xi_{t}\right)_{x}=0, \\
& \left(\xi h \frac{f_{x}}{f}-\xi h_{x}+\frac{1}{n} h(1-m)\left(2 \xi_{x}+\xi \frac{f_{x}}{f}\right)\right) u^{m}+\frac{2}{n}(1-n-m) f h \xi_{t} u^{m-n} \\
& -\frac{8}{n^{2}}(1-n) f^{4}\left(\xi_{t}\right)^{3} u^{1-3 n}+\frac{8}{n^{2}}(1-n)\left[f^{2} \xi_{t}\left(f \xi_{t}\right)_{t}-f^{3} \xi_{t}^{2}\left(2 \xi_{x}+\xi \frac{f_{x}}{f}\right)\right] u^{1-2 n} \\
& +\left[\frac{2}{n} f\left(f \xi_{t}\right)_{t t}-\frac{4}{n} f \xi_{t}\left(f \xi_{t}\right)_{x}+\frac{4}{n^{2}} f^{2} \xi_{t}\left(2 \xi_{x}+\xi \frac{f_{x}}{f}\right)_{t}-\frac{2}{n^{2}}(1-n) f^{2} \xi_{t}\left(2 \xi_{x}+\xi \frac{f_{x}}{f}\right)^{2}\right. \\
& \left.+\frac{4}{n^{2}}(1-n) f\left(f \xi_{t}\right)_{t}\left(2 \xi_{x}+\xi \frac{f_{x}}{f}\right)\right] u^{1-n}+\left[\frac{2}{n^{2}} f\left(2 \xi_{x}+\xi \frac{f_{x}}{f}\right)\left(2 \xi_{x}+\xi_{\frac{x_{x}}{f}}\right)_{t}\right. \\
& \left.+\frac{1}{n} f\left(2 \xi_{x}+\xi \frac{f_{x}}{f}\right)_{t t}-\frac{2}{n} f \xi_{t}\left(2 \xi_{x}+\xi_{\frac{f_{x}}{f}}\right)_{x}-\frac{2}{n}\left(f \xi_{t}\right)_{x x}\right] u-\frac{1}{n}\left(2 \xi_{x}+\xi_{\frac{x}{x}}\right)_{x x} u^{n+1}=0 .
\end{aligned}
$$

Solving the above system with respect to the coefficient functions $\xi$, $f$ and $h$ under the equivalence group $G_{1}^{\sim}$ of the class (4), which consists of the transformations (see Theorem 3 and 4 in [25] for more details): for $n \neq-1$ :

$$
\begin{aligned}
& \tilde{t}=\epsilon_{1} t+\epsilon_{2}, \quad \tilde{x}=\frac{\epsilon_{3} x+\epsilon_{4}}{\epsilon_{5} x+\epsilon_{6}}=: X(x), \quad \tilde{u}=\epsilon_{7} X_{x}^{\frac{1}{2 n+2}} u, \\
& \tilde{f}=\epsilon_{1}^{2} \epsilon_{7}^{n} X_{x}^{-\frac{3 n+4}{2 n+2}} f, \quad \tilde{h}=\epsilon_{7}^{-m+n+1} X_{x}^{-\frac{m+3 n+3}{2 n+2}} h, \quad \tilde{n}=n, \quad \tilde{m}=m,
\end{aligned}
$$

where $\epsilon_{j}(j=1, \ldots, 7)$ are arbitrary constants, $\epsilon_{1} \epsilon_{7} \neq 0, \epsilon_{3} \epsilon_{6}-\epsilon_{4} \epsilon_{5}= \pm 1$, and for $n=-1$ :

$$
\begin{aligned}
& \tilde{t}=\epsilon_{1} t+\epsilon_{2}, \quad \tilde{x}=\epsilon_{3} x+\epsilon_{4}, \quad \tilde{u}=\epsilon_{5} e^{\epsilon_{6} x} u, \\
& \tilde{f}=\epsilon_{1}^{2} \epsilon_{3}^{-2} \epsilon_{5}^{-1} e^{-\epsilon_{6} x} f, \quad \tilde{h}=\epsilon_{3}^{-2} \epsilon_{5}^{-m} e^{-m \epsilon_{6} x} h, \quad \tilde{n}=n, \quad \tilde{m}=m,
\end{aligned}
$$

where $\epsilon_{j}(j=1, \ldots, 6)$ are arbitrary constants, $\epsilon_{1} \epsilon_{3} \epsilon_{5} \neq 0$; we can get a classification of the regular reduction operator for the class (4). It is easy to know that some of the regular reduction operator are equivalent to Lie symmetry operators, which have been given in [25], while some of them are nontrivial. Below, we give a detailed investigations for these cases.

In fact, the first three equations of System (11) imply that there are two cases that should be considered: $n \neq 1$ or not. (It should be noted that $\xi=0$ should be exclude from the consideration because it leads to $\eta=0$ ).

Case 1: $n \neq 1$. In this case, we have $\xi_{t}=0$, and System (11) can be reduced to:

$$
\begin{aligned}
& (3 n+4) \xi_{x x}+2(n+1)\left(\xi \frac{f_{x}}{f}\right)_{x}=0 \\
& \left(\xi h \frac{f_{x}}{f}-\xi h_{x}+\frac{1}{n}(1-m) h\left(2 \xi_{x}+\xi \frac{f_{x}}{f}\right)\right) u^{m}-\frac{1}{n}\left(2 \xi_{x}+\xi \frac{f_{x}}{f}\right)_{x x} u^{n+1}=0
\end{aligned}
$$

Thus, there are two cases that should be considered: $m \neq n+1$ or not. 
Case 1.1: For $m \neq n+1$, from the second Equation of (12), we obtain:

$$
\xi h \frac{f_{x}}{f}-\xi h_{x}+\frac{1}{n}(1-m) h\left(2 \xi_{x}+\xi \frac{f_{x}}{f}\right)=0, \quad\left(2 \xi_{x}+\xi \frac{f_{x}}{f}\right)_{x x}=0 .
$$

Because $\xi_{t}=0$ and $f$ is a function of $x$, the first Equation of (12) suggests that $(3 n+4) \xi_{x}+2(n+1) \xi \frac{f_{x}}{f}$ is independent of the variables $x$ and $t$. Thus, there exists a constant $r$, such that $(3 n+4) \xi_{x}+2(n+1) \xi \frac{f_{x}}{f}=n r$. The second Equation of (13) suggests that there exist two constants $a$ and $b$, such that $2 \xi_{x}+\xi \frac{f_{x}}{f}=n a x+n b$. By solving the last two equations, we obtain:

$$
\xi_{x}=2(n+1)(a x+b)-r, \xi \frac{f_{x}}{f}=2 r-(3 n+4)(a x+b),
$$

which together with the first Equation of (13) imply:

$$
\begin{aligned}
& \xi=a(n+1) x^{2}+[2 b(n+1)-r] x+s, \\
& f(x)=\exp \left(\int \frac{2 r-(3 n+4)(a x+b)}{a(n+1) x^{2}+[2 b(n+1)-r] x+s} \mathrm{~d} x\right), \\
& h(x)=\exp \left(\int \frac{2 r-(m+3 n+3)(a x+b)}{a(n+1) x^{2}+[2 b(n+1)-r] x+s} \mathrm{~d} x\right),
\end{aligned}
$$

where $a, b, r, s$ are arbitrary constants. Thus, the corresponding regular reduction operator has the form:

$$
Q=\partial_{t}+\left[a(n+1) x^{2}+(2 b(n+1)-r) x+s\right] \partial_{x}+(a x+b) u \partial_{u}
$$

which is equivalent to the Lie symmetry operator.

Case 1.2: $m=n+1$. In this case, System (12) can be rewritten as:

$$
(3 n+4) \xi_{x x}+2(n+1)\left(\xi \frac{f_{x}}{f}\right)_{x}=0, \quad \xi h_{x}+2 h \xi_{x}+\frac{1}{n}\left(2 \xi_{x}+\xi \frac{f_{x}}{f}\right)_{x x}=0 .
$$

Integrating these two equations with respect to functions $f(x)$ and $h(x)$, we can obtain:

$$
f(x)=|\xi|^{-\frac{3 n+4}{2 n+2}} \exp \left(r \int \frac{1}{\xi} \mathrm{d} x\right), \quad h(x)=\frac{\xi_{x}^{2}-2 \xi \xi_{x x}-p}{4(n+1) \xi^{2}} .
$$

where $p, r$ are arbitrary constants, $\xi$ is an arbitrary smooth function and $n \neq-1$. In addition, $\eta=\frac{1}{n}\left(2 \xi_{x}+\xi \frac{f_{x}}{f}\right) u=\left(\frac{r}{n}+\frac{\xi_{x}}{2 n+2}\right) u$. Thus, we have a nontrivial regular reduction operator:

$$
Q=\partial_{t}+\xi(x) \partial_{x}+\left[\left(\frac{r}{n}+\frac{\xi_{x}}{2 n+2}\right) u\right] \partial_{u}, \quad n \neq-1 .
$$

It should be noted that the reduction operator for $n=-1$ is also equivalent to the Lie symmetry operator.

Case 2: $n=1$. In this case, we have $\eta=2 f \xi_{t}+\left(2 \xi_{x}+\xi \frac{f_{x}}{f}\right) u$. Thus, System (11) can be reduced to:

$$
\begin{aligned}
& \left(7 \xi_{x}+4 \xi \frac{f_{x}}{f}\right)_{x}=0, \quad 2\left[\xi_{x}+2(\xi+1) \frac{f_{x}}{f}\right] \xi_{t}+4(\xi+1) \xi_{t x}+\xi_{t t}=0, \\
& {\left[2 f\left(2 \xi_{x}+\xi \frac{f_{x}}{f}\right)\left(2 \xi_{x}+\xi \frac{f_{x}}{f}\right)_{t}+f\left(2 \xi_{x}+\xi \frac{f_{x}}{f}\right)_{t t}-2 f \xi_{t}\left(2 \xi_{x}+\xi \frac{f_{x}}{f}\right)_{x}-2\left(f \xi_{t}\right)_{x x}\right] u} \\
& -\left(2 \xi_{x}+\xi \frac{f_{x}}{f}\right)_{x x} u^{2}+2 f^{2}\left(2 \xi_{t} \xi_{t x}+\xi_{t t t}\right)+\left[\xi h \frac{f_{x}}{f}-\xi h_{x}+(1-m) h\left(2 \xi_{x}+\xi \frac{f_{x}}{f}\right)\right] u^{m} \\
& -2 m h f \xi_{t} u^{m-1}=0 .
\end{aligned}
$$

Comparing different powers of $u$, we conclude that five cases should be considered, that is (i) Case 2.1: $m=0$; (ii) case 2.2: $m=1$; (iii) Case 2.3: $m=2$; (iv) Case 2.4: $m=3$ and (v) Case 2.5: 
$m \neq 0,1,2,3$. Below, we mainly give a detailed discussion for Cases 2.2 and 2.3, and the other cases can be considered in a similar way.

Case 2.2: When $m=1$, the third Equation of (17) implies:

$$
\begin{aligned}
& \xi h \frac{f_{x}}{f}-\xi h_{x}+f\left(2 \xi_{x}+\xi \frac{f_{x}}{f}\right)_{t t}+2 f\left(2 \xi_{x}+\xi \frac{f_{x}}{f}\right)\left(2 \xi_{x}+\xi \frac{f_{x}}{f}\right)_{t} \\
& -2 f \xi_{t}\left(2 \xi_{x}+\xi \frac{f_{x}}{f}\right)_{x}-2\left(f \xi_{t}\right)_{x x}=0 \\
& 2 f \xi_{t} \xi_{t x}+f \xi_{t t t}-h \xi_{t}=0, \quad\left(2 \xi_{x}+\xi \frac{f_{x}}{f}\right)_{x x}=0 .
\end{aligned}
$$

From the last equation of System (18), we can know that there exist two functions $a(t)$ and $b(t)$, such that $2 \xi_{x}+\xi \frac{f_{x}}{f}=a(t) x+b(t)$. On the other hand, the first Equation of (17) implies that there exists a function $c(t)$, such that $7 \xi_{x}+4 \frac{f_{x}}{f}=c(t)$. Solving the last two equations gives:

$$
\xi_{x}=4 a(t) x+4 b(t)-c(t), \quad \xi \frac{f_{x}}{f}=-7 a(t) x-7 b(t)+2 c(t),
$$

from which we can get:

$$
\xi=2 a(t) x^{2}+4 b(t) x-c(t) x+d(t), \quad f(x)=\exp \left(\int \frac{-7 a(t) x-7 b(t)+2 c(t)}{2 a(t) x^{2}+4 b(t) x-c(t) x+d(t)} \mathrm{d} x\right),
$$

where $d(t)$ is an arbitrary function. Since $\frac{f_{x}}{f}$ is independent of $t$, we see that:

$$
\left[\frac{-7 a(t) x-7 b(t)+2 c(t)}{2 a(t) x^{2}+4 b(t) x-c(t) x+d(t)}\right]_{t}=0
$$

which leads to:

$$
\left\{\begin{array}{l}
14\left[a(t) b^{\prime}(t)-a^{\prime}(t) b(t)\right]+3\left[a^{\prime}(t) c(t)-a(t) c^{\prime}(t)\right]=0 \\
{\left[b(t) c^{\prime}(t)-b^{\prime}(t) c(t)\right]+7\left[a(t) d^{\prime}(t)-a^{\prime}(t) d(t)\right]=0} \\
2\left[c^{\prime}(t) d(t)-c(t) d^{\prime}(t)\right]+7\left[b(t) d^{\prime}(t)-b^{\prime}(t) d(t)\right]=0 .
\end{array}\right.
$$

Now, we multiply both sides of the second Equation of (17) by $\xi$ and substitute (19) into it, then simplify the equation and compare the coefficient of $x^{i}(i=0,1, \ldots, 5)$ to obtain:

$$
\left\{\begin{array}{l}
a^{\prime}(t)=0, \quad a^{2}\left[-4 b^{\prime}(t)+c^{\prime}(t)\right]=0, \\
a\left[8 c(t) c^{\prime}(t)+20 a d^{\prime}(t)+c^{\prime \prime}(t)-4 b^{\prime \prime}(t)-32 c(t) b^{\prime}(t)+112 b(t) b^{\prime}(t)-28 b(t) c^{\prime}(t)\right]=0, \\
-4 c^{\prime \prime}(t) b(t)+32 a c(t) d^{\prime}(t)-80 a b^{\prime}(t)+20 a c^{\prime}(t)-4 b^{\prime \prime}(t) c(t)-120 a b(t) d^{\prime}(t) \\
+c^{\prime \prime}(t) c(t)+2 d^{\prime \prime}(t) a+2 c^{2}(t) c^{\prime}(t)-12 b(t) c(t) c^{\prime}(t)+16 b(t)^{2} c^{\prime}(t)-8 c^{2}(t) b^{\prime}(t) \\
+48 b(t) c(t) b^{\prime}(t)+16 b^{\prime \prime}(t) b(t)-64 b^{2}(t) b^{\prime}(t)-16 a d(t) b^{\prime}(t)+4 a d(t) c^{\prime}(t)=0, \\
{\left[-20 a d^{\prime}(t)+48 b(t) b^{\prime}(t)+2 c(t) c^{\prime}(t)-12 b(t) c^{\prime}(t)-8 c(t) b^{\prime}(t)+4 b^{\prime \prime}(t)-c^{\prime \prime}(t)\right] d(t)} \\
-80 b^{2}(t) d^{\prime}(t)+4 d^{\prime \prime}(t) b(t)-d^{\prime \prime}(t) c(t)-4 c(t) c^{\prime}(t)+44 b(t) c(t) d^{\prime}(t)-28 a d^{\prime}(t) \\
-48 b(t) b^{\prime}(t)-6 c^{2}(t) d^{\prime}(t)+12 b(t) c^{\prime}(t)+16 c(t) b^{\prime}(t)=0, \\
{\left[16 b^{\prime}(t)-4 c^{\prime}(t)\right] d^{2}(t)+\left[-20 b(t) d^{\prime}(t)-4 c^{\prime}(t)+6 c(t) d^{\prime}(t)+d^{\prime \prime}(t)+16 b^{\prime}(t)\right] d(t)} \\
+8 c(t) d^{\prime}(t)-28 b(t) d^{\prime}(t)=0 .
\end{array}\right.
$$

Note that $\xi$ is assumed not to be identical with zero; thus, we find that Systems (20) and (21) can be reduced to:

$$
a^{\prime}(t)=0, \quad b^{\prime}(t)=0, \quad c^{\prime}(t)=0, \quad d^{\prime}(t)=0
$$


or:

$$
a=0, \quad c(t)=4 b(t), \quad d(t)=q b(t), \quad q b^{\prime \prime}(t)+4 q b(t) b^{\prime}(t)+4 b^{\prime}(t)=0
$$

or:

$$
a=0, \quad 2 c(t)=7 b(t), \quad b^{\prime \prime}(t)=-3 b(t) b^{\prime}(t), \quad b(t) d^{\prime}(t)+2 b^{\prime}(t)(d(t)+1)+d^{\prime \prime}(t)=0
$$

or:

$$
a=0, \quad c(t)=3 b(t), \quad d=q b(t), \quad b^{\prime \prime}(t)+2 b(t) b^{\prime}(t)=0,
$$

where $q$ is an arbitrary constant.

Case 2.2a: If System (22) is satisfied, then $\xi_{t}=0$; the second equation of (18) is an identity. The expression (19) can be rewritten as:

$$
\xi=2 a x^{2}+4 b x-c x+d, \quad f(x)=\exp \left(\int \frac{-7 a x-7 b+2 c}{2 a x^{2}+4 b x-c x+d} \mathrm{~d} x\right),
$$

where $a, b, c$ and $d$ are arbitrary constants. The first Equation of (18) is reduced to $h_{x} / h=f_{x} / f$, which leads to $h(x)=\epsilon f(x)(\epsilon= \pm 1) \bmod G_{1}^{\sim}$. In addition, $\eta=2 f \xi_{t}+\left(2 \xi_{x}+\xi \frac{f_{x}}{f}\right) u=(a x+b) u$. Thus, we have:

$$
\begin{aligned}
& \xi=2 a x^{2}+4 b x-c x+d, \quad \eta=(a x+b) u, \\
& f(x)=\exp \left(\int \frac{-7 a x-7 b+2 c}{2 a x^{2}+4 b x-c x+d} \mathrm{~d} x\right), \quad h(x)=\epsilon f(x),
\end{aligned}
$$

where $a, b, c, d$ are arbitrary constants and $\epsilon= \pm 1$. Thus, the corresponding regular reduction operator has the form:

$$
Q=\partial_{t}+\left(2 a x^{2}+4 b x-c x+d\right) \partial_{x}+(a x+b) u \partial_{u},
$$

which is equivalent to the Lie symmetry operator.

Case 2.2b: If System (23) is satisfied, then the expression (19) can be rewritten as:

$$
\xi=q b(t), \quad f(x)=\exp \left(\frac{x}{q}\right)
$$

Hence, $\xi_{x}=0, k=b(t)$. If $b^{\prime}(t)=0$, then $a(t), b(t), c(t), d(t)$ satisfy System (22), and the solution is included in Case 2.2a. We suppose that $b^{\prime}(t) \neq 0$. From the second Equation of (18), we see that $h=f \xi_{t t t} / \xi_{t}$. Substitute it into the first Equation of (18) to get $f k_{t t}+2 f k k_{t}-2 f_{x x} \xi_{t}=0$. Further, it can be reduced to $q b^{\prime \prime}(t)+2 q b(t) b^{\prime}(t)-2 b^{\prime}(t)=0$. Combine it with the fourth Equation of (23) to get $b(t)=-3 / q$, which is a contradiction to the hypothesis $b^{\prime}(t) \neq 0$.

Case 2.2c: If System (24) is satisfied, then the expression (19) can be rewritten as:

$$
\xi=\frac{1}{2} b(t) x+d(t), \quad f(x)=1 \quad \bmod G_{1}^{\sim} .
$$

If $b^{\prime}(t)=d^{\prime}(t)=0$, then $a(t), b(t), c(t), d(t)$ satisfy both Systems (22) and (24), and the solution is included in Case 2.2a. We suppose that $b^{\prime 2}(t)+d^{\prime 2}(t) \neq 0$. Substitute (29) into the first Equation of (18) to obtain:

$$
h_{x}=\frac{2\left[b^{\prime \prime}(t)+2 b(t) b^{\prime \prime}(t)\right]}{b(t) x+2 d(t)} .
$$

Substitute (29) into the second Equation of (18) to obtain:

$$
h(x)=b^{\prime}(t)+\frac{b^{\prime \prime \prime}(t) x+2 d^{\prime \prime \prime}(t)}{b^{\prime}(t) x+2 d^{\prime}(t)} .
$$


Substituting it into (30) yields:

$$
\frac{b^{\prime \prime \prime}(t) d^{\prime}(t)-b^{\prime}(t) d^{\prime \prime \prime}(t)}{\left[b^{\prime}(t) x+2 d^{\prime}(t)\right]^{2}}=\frac{b^{\prime \prime}(t)+2 b(t) b^{\prime}(t)}{b(t) x+2 d(t)} .
$$

Compare the coefficient of $x^{2}$ to obtain $b^{\prime 2}(t)\left[b^{\prime \prime}(t)+2 b(t) b^{\prime}(t)\right]=0$. Substitute the third Equation of (24) into it to obtain $b(t) b^{13}(t)=0$; hence, $b^{\prime}(t)=0$. Thus, the fourth Equation of (24) can be reduced to $b d^{\prime}(t)+d^{\prime \prime}(t)=0$. Solving this linear ordinary differential equation gives $d(t)=\gamma_{1} e^{-b t}+\gamma_{0}$, where $\gamma_{1}$ and $\gamma_{0}$ are two arbitrary constants. Therefore, the expressions (29) and (31) can be rewritten as:

$$
\xi=\frac{1}{2} b x+\gamma_{1} e^{-b t}+\gamma_{0}, \quad f(x)=1, \quad h(x)=b^{2} \quad \bmod G_{1}^{\sim} .
$$

System (18) is verified to be true. In addition, $\eta=2 f \xi_{t}+\left(2 \xi_{x}+\frac{f_{x}}{f}\right) u=b u-2 \gamma_{1} b e^{-b t}$. Therefore, we have:

$$
\xi=\frac{1}{2} b x+\gamma_{1} e^{-b t}+\gamma_{0}, \quad \eta=b u-2 \gamma_{1} b e^{-b t}, \quad f(x)=1, \quad h(x)=b^{2},
$$

where $b, \gamma_{1}, \gamma_{0}$ are arbitrary constants. Thus, we have a nontrivial regular reduction operator:

$$
Q=\partial_{t}+\left(\frac{1}{2} b x+\gamma_{1} e^{-b t}+\gamma_{0}\right) \partial_{x}+\left(b u-2 \gamma_{1} b e^{-b t}\right) \partial_{u}
$$

Case 2.2d: If System (25) is satisfied, then the expression (19) can be rewritten as:

$$
\xi=b(t)(x+q), \quad f(x)=\frac{1}{x+q} \bmod G_{1}^{\sim} .
$$

Substitute it into the first Equation of (18) to obtain $b(t)(x+q)\left[h+(x+q) h_{x}\right]=\left[b^{\prime \prime}(t)+2 b(t) b^{\prime}(t)\right]$. Substitute the fourth Equation of (25) into it to get $b(t)(x+q)\left[h+(x+q) h_{x}\right]=0$. It follows that $h(x)=r /(x+q)$, where $r$ is a nonzero constant. Substitute it and (34) into the second Equation of (18) to obtain $2 b^{\prime 2}(t)+b^{\prime \prime \prime}(t)-r b^{\prime}(t)=0$. From the fourth Equation of (25), we find $b^{\prime \prime \prime}(t)=4 b^{2}(t) b^{\prime}(t)-2 b^{\prime 2}(t)$. Substitute it into the preceding equation to get $b^{\prime}(t)\left[4 b^{2}(t)-r\right]=0$, which leads to $b^{\prime}(t)=0$. Then, $a(t), b(t), c(t), d(t)$ satisfy System (22), and the solution is included in Case 2.2a.

Case 2.3: When $m=2$, System (17) implies:

$$
\begin{aligned}
& \left(7 \xi_{x}+4 \xi_{\frac{f_{x}}{f}}\right)_{x}=0, \quad 2\left(\xi_{x}+2 \xi \frac{f_{x}}{f}\right) \xi_{t}+4 \xi_{t} \frac{f_{x}}{f}+4 \xi \xi_{t x}+4 \xi_{t x}+\xi_{t t}=0, \\
& 2 \xi_{t} \xi_{t x}+\xi_{t t t}=0, \quad \xi h_{x}+2 h \xi_{x}+\left(2 \xi_{x}+\xi \frac{f_{x}}{f}\right)_{x x}=0, \\
& 2 f\left(2 \xi_{x}+\xi \frac{f_{x}}{f}\right)\left(2 \xi_{x}+\xi \frac{f_{x}}{f}\right)_{t}+f\left(2 \xi_{x}+\xi \frac{f_{x}}{f}\right)_{t t}-4 h f \xi_{t}-2 f \xi_{t}\left(2 \xi_{x}+\xi \frac{f_{x}}{f}\right)_{x}-2\left(f \xi_{t}\right)_{x x}=0 .
\end{aligned}
$$

From the first and the fourth equation of System (35), we can get:

$$
f(x)=|\xi|^{-7 / 4} \exp \left(\alpha(t) \int \frac{\mathrm{d} x}{\xi}\right), \quad h(x)=\frac{\xi_{x}^{2}-2 \xi \xi_{x x}+q}{8 \xi^{2}}
$$


where $\alpha(t)$ is an arbitrary function and $q$ is a constant. Substituting these expressions into the rest of the equations of System (35), we can see that $\xi(t, x)$ and $\alpha(t)$ satisfy the overdetermined system of partial differential equations:

$$
\begin{aligned}
& 2 \xi_{t} \xi_{t x}+\xi_{t t t}=0, \quad \xi_{t t}-3 \xi_{t x}-5 \xi_{t} \xi_{x}+4 \xi_{t x}+4 \alpha \xi_{t}+4 \alpha_{t}=0, \\
& 2 \xi^{2}\left(\frac{1}{4} \xi_{x}+\alpha\right)_{t t}+2 \xi^{2}\left[\left(\frac{1}{4} \xi_{x}+\alpha\right)^{2}\right]_{t}-\xi^{2} \xi_{t} \xi_{x x}-\xi_{t}\left(\xi_{x}^{2}-2 \xi \xi_{x x}+q\right) \\
& -4\left[\left(\alpha_{t}-\frac{3}{4} \xi_{t x}\right)\left(\alpha-\frac{7}{4} \xi_{x}\right) \xi-\frac{3}{4} \xi^{2} \xi_{t x x}\right]=0 .
\end{aligned}
$$

In addition, we have:

$$
\eta=2 f \xi_{t}+\left(2 \xi_{x}+\xi \frac{f_{x}}{f}\right) u=2 \xi_{t}|\xi|^{-7 / 4} \exp \left(\alpha(t) \int \frac{\mathrm{d} x}{\xi}\right)+\left[\frac{1}{4} \xi_{x}+\alpha(t)\right] u .
$$

Thus, we have a nontrivial regular reduction operator:

$$
Q=\partial_{t}+\xi(t, x) \partial_{x}+\left\{2 \xi_{t}|\xi|^{-7 / 4} \exp \left(\alpha(t) \int \frac{\mathrm{d} x}{\xi}\right)+\left[\frac{1}{4} \xi_{x}+\alpha(t)\right] u\right\} \partial_{u}
$$

where $\xi(t, x)$ and $\alpha(t)$ satisfy the overdetermined system of partial differential equations (36).

In particular, if $\xi_{t}=0$, from System (35), we can obtain:

$$
\xi=\xi(x), \quad \eta=\frac{1}{4}\left(\xi_{x}+a\right) u, \quad f(x)=|\xi|^{-7 / 4} \exp \left(\frac{a}{4} \int \frac{\mathrm{d} x}{\xi}\right), \quad h(x)=\frac{\xi_{x}^{2}-2 \xi \xi x x+q}{8 \xi^{2}} .
$$

where $a, q$ are arbitrary constants. Thus, we have a nontrivial regular reduction operator:

$$
Q=\partial_{t}+\xi(x) \partial_{x}+\left(\frac{1}{4} \xi_{x}+a\right) u \partial_{u}
$$

which is equivalent to Operator (16) with $n=1$. Therefore, this special case can be included in Case 1.2, and we can impose an additional constraint $\xi_{t} \neq 0$ on the regular reduction operator (37).

Remark 2. It should be noted that we can also give detailed analysis for Cases 2.1, 2.4 and 2.5 in a way similar to Cases 2.2 and 2.3. However, we find that all of the regular reduction operators resulting from these Cases 2.1, 2.4 and 2.5 are trivial, which are equivalent to Lie symmetry operators. Thus, we omit these results in order to avoid tediousness.

From the above discussion, we can arrive at the following two theorems.

Theorem 3. A complete list of $G_{1}^{\sim}$-inequivalent Equation (3) having a nontrivial regular reduction operator is exhausted by the ones given in Table 1.

Table 1. Results of regular reduction operator classification of Class (3).

\begin{tabular}{cccccc}
\hline $\mathbf{N}$ & $n$ & $\boldsymbol{m}$ & $f(x)$ & $h(x)$ & Regular Reduction Operator $Q$ \\
\hline 1 & $\neq-1$ & $n+1$ & $|\xi|^{-\frac{3 n+4}{2 n+2}} \exp \left(r \int \frac{1}{\xi} \mathrm{d} x\right)$ & $\frac{\xi_{x}^{2}-2 \xi \xi_{x x}-p}{4(n+1) \xi^{2}}$ & $\partial_{t}+\xi(x) \partial_{x}+\left(\frac{r}{n}+\frac{\xi_{x}}{2 n+2}\right) u \partial_{u}$ \\
\hline 2 & 1 & 1 & 1 & $b^{2}$ & $\partial_{t}+\left(\frac{1}{2} b x+\gamma_{1} e^{-b t}+\gamma_{0}\right) \partial_{x}+\left(b u-2 \gamma_{1} b e^{-b t}\right) \partial_{u}$ \\
\hline 3 & 1 & 2 & $|\xi|^{-\frac{7}{4}} \exp \left(\alpha(t) \int \frac{\mathrm{d} x}{\zeta}\right)$ & $\frac{\xi_{x}^{2}-2 \xi \xi_{x x}+q}{8 \xi^{2}}$ & $\partial_{t}+\xi(t, x) \partial_{x}+\left\{\left.2 \xi t \xi\right|^{-\frac{7}{4}} \exp \left(\alpha(t) \int \frac{\mathrm{d} x}{\xi}\right)\right.$ \\
& & & & $\left.+\left[\frac{1}{4} \xi_{x}+\alpha(t)\right] u\right\} \partial_{u}$ \\
\hline
\end{tabular}

Here $r, p, b, \gamma_{1}, \gamma_{0}$ are arbitrary constants; $b \neq 0, \xi(x)$ in Case 2.1 is an arbitrary functions of the variables $x$, $\xi(t, x)$; and $\alpha(t)$ in Case 2.3 satisfy the overdetermined system of partial differential Equation (36) and $\xi_{t} \neq 0$. 
Theorem 4. Any reduction operator of an equations from Class (3) having the form (10) with $\xi_{t}=0, \xi_{x x x}=0$ is equivalent to a Lie symmetry operator of this equation.

\section{Exact Solutions}

In this section, we construct the nonclassical reduction and exact solutions for the classification models in Table 1 by using the corresponding regular reduction operator. Lie reduction and exact solutions of the equation from Class (3) have been investigated in some literature works; see, for example, References $[9,15,16,18,21,24,25]$. We choose Case 1 in Table 1 as an example to implement the reduction; Case 3 can be considered in a similar way. While for Case 2, the nonclassical reduction and exact solution of the corresponding equation $u_{t t}=\left(u u_{x}\right)_{x}+b^{2} u$ have been investigated by Fushchich et al. in [34,47]; thus, we do not consider it here.

For the first case in Table 1, the corresponding equation is:

$$
\left[|\xi|^{-\frac{3 n+4}{2 n+2}} \exp \left(r \int \frac{1}{\xi} \mathrm{d} x\right)\right] u_{t t}-\left(u^{n} u_{x}\right)_{x}-\frac{\xi_{x}^{2}-2 \xi \xi x x-p}{4(n+1) \xi^{2}} u^{n+1}=0,
$$

which admits the regular reduction operator:

$$
Q=\partial_{t}+\xi(x) \partial_{x}+\left(\frac{r}{n}+\frac{\xi_{x}}{2 n+2}\right) u \partial_{u}
$$

An ansatz constructed by this operator has the form:

$$
u(t, x)=\varphi(\omega)|\xi|^{\frac{1}{2 n+2}} \exp \left(\frac{r}{n} \int \frac{1}{\xi} \mathrm{d} x\right), \quad \text { where } \omega=t-\int \frac{1}{\xi} \mathrm{d} x .
$$

Substituting this ansatz into Equation (40) leads to the reduced ODE:

$$
\begin{aligned}
& {\left[\left(4 r^{2}-p\right) n^{2}+4(2 n+1) r^{2}\right] \varphi^{n+1}(\omega)+4 n(n+1)\left[n \varphi^{\prime \prime}(\omega)-2(n+1) r \varphi^{\prime}(\omega)\right] \varphi^{n}(\omega)} \\
& +4 n^{3}(n+1) \varphi^{\prime 2}(\omega) \varphi^{n-1}(\omega)-4 n^{2}(n+1) \varphi^{\prime \prime}(\omega)=0
\end{aligned}
$$

Because there are higher nonlinear terms, we were not able to completely solve the above equation. Thus, we try to solve this equation under different additional constraints imposed on $p$ and $r$.

We first rewrite Equation (41) as:

$$
\begin{aligned}
& 4 n^{2}(n+1)\left[\varphi^{\prime}(\omega) \varphi^{n}(\omega)\right]^{\prime}-4 n^{2}(n+1) \varphi^{\prime \prime}(\omega)-8 n(n+1)^{2} r \varphi^{\prime}(\omega) \varphi^{n}(\omega) \\
& +\left[4(n+1)^{2} r^{2}-p n^{2}\right] \varphi^{n+1}(\omega)=0 .
\end{aligned}
$$

If we take $p=4\left(1+\frac{1}{n}\right)^{2} r^{2}$, then the general solution of (42) can be written in the implicit form:

$$
\int \frac{n\left(\varphi^{n}-1\right)}{2 r \varphi^{n+1}+c_{1}} \mathrm{~d} \varphi=\omega+c_{2}
$$

Up to the similarity of solutions of Equation (3), the constant $c_{2}$ is inessential and can be set to equal zero by a translation of $\omega$, which is always induced by a translation of $t$.

If we further set $n=1$, the general solution (43) can be rewritten in the following implicit forms, that is if $\frac{c_{1}}{2 r}>0$, then we have:

$$
\frac{1}{4 r} \ln \left|2 r \varphi^{2}+c_{1}\right|-\frac{\arctan \left(\sqrt{\frac{2 r}{c_{1}}} \varphi\right)}{\sqrt{2 r c_{1}}}+C=\omega,
$$


while if $\frac{c_{1}}{2 r}<0$, then we have:

$$
\frac{1}{4 r} \ln \left|2 r \varphi^{2}+c_{1}\right|-\frac{\operatorname{arctanh}\left(\sqrt{-\frac{2 r}{c_{1}}} \varphi\right)}{\sqrt{-2 r c_{1}}}+C=\omega ;
$$

and if $c_{1}=0$ and $r \neq 0$, then we have:

$$
\frac{1}{2 r}\left(\ln |\varphi|+\varphi^{-1}\right)+C=\omega,
$$

while if $c_{1} \neq 0$ and $r=0$, then we have:

$$
\frac{1}{c_{1}}\left(\frac{1}{2} \varphi^{2}-\varphi\right)+C=\omega
$$

where $C$ is an arbitrary constant.

Thus, we obtain the following solution:

$$
u(t, x)=\varphi(\omega)|\xi|^{\frac{1}{4}} \exp \left(r \int \frac{1}{\xi} \mathrm{d} x\right), \quad \omega=t-\int \frac{1}{\xi} \mathrm{d} x
$$

for the equation:

$$
\left[|\xi|^{-\frac{7}{4}} \exp \left(r \int \frac{1}{\xi} \mathrm{d} x\right)\right] u_{t t}-\left(u u_{x}\right)_{x}-\frac{\xi_{x}^{2}-2 \xi \xi_{x x}-8 r^{2}}{8 \xi^{2}} u^{2}=0,
$$

where $\varphi$ satisfies Equations (44)-(47), $\xi$ is an arbitrary function and $r$ is a non-zero constant.

If we further set $r=0$, the general solution (43) can be rewritten in the implicit form:

$$
\frac{1}{n+1} \varphi^{n+1}(\omega)-\varphi(\omega)=c_{1} \omega+c_{2}
$$

Thus, we obtain the following solution:

$$
u(t, x)=\varphi(\omega) \mid \xi^{\frac{1}{2 n+2}}, \quad \omega=t-\int \frac{1}{\xi} \mathrm{d} x
$$

for the equation:

$$
|\xi|^{-\frac{3 n+4}{2 n+2}} u_{t t}-\left(u^{n} u_{x}\right)_{x}-\frac{\xi_{x}^{2}-2 \xi \xi_{x x}}{4(n+1) \xi^{2}} u^{n+1}=0,
$$

where $\varphi$ satisfies Equation (48) and $\xi$ is an arbitrary function. In particular, when $n=1$, Equation (48) is reduced to Equation (47); thus, we have:

$$
\varphi(\omega)=1 \pm \sqrt{1+2\left(c_{1} \omega-C\right)} .
$$

Thus, we obtain an explicit solution:

$$
u(t, x)=\left[1 \pm \sqrt{1+2\left(c_{1} \omega-C\right)}\right]|\xi|^{\frac{1}{4}}, \quad \omega=t-\int \frac{1}{\xi} \mathrm{d} x
$$

for the equation:

$$
|\xi|^{-\frac{7}{4}} u_{t t}-\left(u u_{x}\right)_{x}-\frac{\xi_{x}^{2}-2 \xi \xi \xi_{x x}}{8 \xi^{2}} u^{2}=0 .
$$

If we take different functions for $\xi$, then we can obtain a series of solutions for the corresponding equations. In order to avoid tediousness, we do not make a further discussion here. 


\section{Conclusions and Discussion}

In this paper, we have given a detailed investigation of the reduction operators of the variable coefficient nonlinear wave equations (1) (equivalently to (3)) by using the singular reduction operator theory. A classification of regular reduction operators is performed with respect to generalized extended equivalence groups. The main results on classification for Equation (3) are collected in Table 1, where we list three inequivalent cases with the corresponding regular reduction operators. The nonclassical symmetry reduction of a class of nonlinear wave Model (40), which singled out the classification models, is also performed. These are utilized to construct some non-Lie exact solutions, which are invariant under certain conditional symmetries for the corresponding model.

The nonclassical symmetry analysis of the class of hyperbolic type nonlinear wave Equation (1) presented in this paper is preliminary. In fact, it is easy to know that the well-known wave equation $u_{t t}=u u_{x x}$ possesses much wider Q-conditional (nonclassical) symmetry, which includes 12 nontrivial reduction operators (see Chapter 7 in [34] for details). Therefore, for Equation (1), we may find more reduction operators besides the ones given in Table 1. One possible way is that we can try to present a particular solution of (8) leading to a Q-conditional symmetry. However, Equation (8) is highly nonlinear, and it is difficult to solve; hence, we do not discuss it in this paper. Another way is to consider the special case $\tau=0$, which is also excluded from our above discussion. Further studies along these lines may lead to more reduction operators. Furthermore, other different properties of Equation (1), including nonclassical potential symmetries, nonclassical potential exact solutions and physical application, will also be investigated in subsequent publications.

Acknowledgments: This work was partially supported by the National Natural Science Foundation of China (Grant No. 11501204), the Natural Science Foundation of Shanghai (Grant No. 15ZR1408300) and the Shanghai Key Laboratory of Intelligent Information Processing (Grant No. IIPL-2014-001).

Author Contributions: All the authors Dingjiang Huang, Yan Zhu and Qinmin Yang make contributions to Section 2.2. Dingjiang Huang make contribution to Sections 1, 2.1, 3 and 4.

Conflicts of Interest: The authors declare no conflict of interest.

\section{References}

1. Ablowitz, M.J.; Clarkson, P.A. Solitons, Nonlinear Evolution Equations and Inverse Scattering; Cambridge University Press: Cambridge, UK, 1991.

2. Rogers, C.; Schief, W.K. Bäcklund and Darboux Transformations, Geometry and Morden Applications in Soliton Theory; Cambridge University Press: Cambridge, UK, 2002.

3. Roy-Chowdhury, A.K. Painleve Analysis and Its Applications; Chapman and Hall/CRC Press: Boca Raton, FL, USA, 1999.

4. Ablowitz, M.J.; Musslimani, Z.H. Integrable nonlocal nonlinear Schrodinger equation. Phys. Rev. Lett. 2013, $110,064105$.

5. Ablowitz, M.J.; Musslimani, Z.H. Inverse scattering transform for the integrable nonlocal nonlinear Schrodinger equations. Nonlinearity 2016, 29, 915-946.

6. Olver, P.J. Application of Lie Groups to Differential Equations; Springer: New York, NY, USA, 1986.

7. Bluman, G.W.; Kumei, S. Symmetries and Differential Equations; Springer: Berlin, Germany, 1989.

8. Ames, W.F. Nonlinear Partial Differential Equations in Engineering; Academic: New York, NY, USA, 1972; Volume II, pp. 50-52.

9. Ames, W.F.; Adams, E.; Lohner, R.G. Group properties of $u_{t t}=\left[f(u) u_{x}\right]_{x}$. Int. J. Non-Linear Mech. 1981, 16, 439-447.

10. Ibragimov, N.H. (Ed.) Lie Group Analysis of Differential Equations-Symmetries, Exact Solutions and Conservation Laws; CRC Press: Boca Raton, FL, USA, 1994; Volume 1.

11. Arrigo, D.J. Group properties of $u_{x x}-u_{y}^{m} u_{y y}=f(u)$. Int. J. Non-Linear Mech. 1991, 26, 619-629.

12. Bluman, G.W.; Cheviakov, A.F. Nonlocally related systems, linearization and nonlocal symmetries for the nonlinear wave equation. J. Math. Anal. Appl. 2007, 333, 93-111. 
13. Bluman, G.W.; Temuerchaolu; Sahadevan, R. Local and nonlocal symmetries for nonlinear telegraph equation. J. Math. Phys. 2005, 46, 023505.

14. Chikwendu, S.C. Non-linear wave propagation solutions by Fourier transform perturbation. Int. J. Non-Linear Mech. 1981, 16 117-128.

15. Donato, A. Similarity analysis and nonlinear wave propagation. Int. J. Non-Linear Mech. 1987, 22 307-314.

16. Gandarias, M.L.; Torrisi, M.; Valenti, A. Symmetry classification and optimal systems of a non-linear wave equation. Int. J. Non-Linear Mech. 2004, 39, 389-398.

17. Huang, D.J.; Ivanova, N.M. Group analysis and exact solutions of a class of variable coefficient nonlinear telegraph equations. J. Math. Phys. 2007, 48, 073507.

18. Huang, D.J.; Zhou, S.G. Group properties of generalized quasi-linear wave equations. J. Math. Anal. Appl. 2010, 366, 460-472.

19. Huang, D.J.; Zhou, S.G. Group-theoretical analysis of variable coefficient nonlinear telegraph equations. Acta Appl. Math. 2012, 117, 135-183.

20. Ibragimov, N.H.; Torrisi, M.; Valenti, A. Preliminary group classification of equations $v_{t t}=f\left(x, v_{x}\right) v_{x x}+$ $g\left(x, v_{x}\right)$. J. Math. Phys. 1991, 32, 2988-2995.

21. Oron, A.; Rosenau, P. Some symmetries of the nonlinear heat and wave equations. Phys. Lett. A 1986, 118, 172-176.

22. Pucci, E. Group analysis of the equation $u_{t t}+\lambda u_{x x}=g\left(u, u_{x}\right)$. Riv. Mat. Univ. Parma 1987, 12, 71-87.

23. Pucci, E.; Salvatori, M.C. Group properties of a class of semilinear hyperbolic equations. Int. J. Non-Linear Mech. 1986, 21, 147-155.

24. Torrisi, M.; Valenti, A. Group properties and invariant solutions for infinitesimal transformations of a nonlinear wave equation. Int. J. Non-Linear Mech. 1985, 20, 135-144.

25. Huang, D.J.; Yang, Q.M.; Zhou, S.G. Lie symmetry classification and equivalence transformation of variable coefficient nonlinear wave equations with power nonlinearities. Chin. J. Contemp. Math. 2012, 33, 205-214.

26. Huang, D.J.; Yang, Q.M.; Zhou, S.G. Conservation law classification of variable coefficient nonlinear wave equation with power Nonlinearity. Chin. Phys. B 2011, 20, 070202.

27. Huang, D.J.; Ivanova, N.M. Algorithmic framework for group analysis of differential equations and its application to generalized Zakharov-Kuznetsov equations. J. Differ. Equ. 2016, 260, 2354-2382.

28. Ovsiannikov, L.V. Group Analysis of Differential Equations; Academic Press: New York, NY, USA, 1982.

29. Bluman, G.W.; Cole, J.D. The general similarity solution of the heat equation. J. Math. Mech. 1969, 18, 1025-1042.

30. Levi, D.; Winternitz, P. Non-classical symmetry reduction: Example of the Boussinesq equation. J. Phys. A Math. Gen. 1989, 22, 2915-2924.

31. Arrigo, D.J.; Broadbridge, P.; Hill, J.M. Nonclassical symmetry solutions and the methods of Bluman-Cole and Clarkson-Kruskal. J. Math. Phys. 1993, 34, 4692-4703.

32. Zhdanov, R.Z.; Tsyfra, I.M.; Popovych, R.O. A precise definition of reduction of partial differential equations. J. Math. Anal. Appl. 1999, 238, 101-123.

33. Olver, P.J.; Rosenau, P. Group-invariant solutions of differential equations. SIAM J. Appl. Math. 1987, 47, 263-278.

34. Fushchych, W.I.; Shtelen, W.M.; Serov, N.I. Symmetry Analysis and Exact Solutions of Nonlinear Equations of Mathematical Physics; Kluwer: Dordrecht, The Netherlands, 1993.

35. Fushchych, W.I.; Shtelen, W.M.; Serov, M.I.; Popovych, R.O. Q-conditional symmetry of the linear heat equation. Proc. Acad. Sci. Ukraine 1992, 12, 28-33.

36. Fushchych, W.I.; Zhdanov, R.Z. Conditional symmetry and reduction of partial differential equations. Ukr. Math. J. 1992, 44, 970-982.

37. Cherniha, R. Conditional symmetries for systems of PDEs: New definitions and their application for reaction-diffusion systems. J. Phys. A Math. Theor. 2010, 43, 405207.

38. Cherniha, R.; Pliukhin, O. New conditional symmetries and exact solutions of reaction-diffusion systems with power diffusivities. J. Phys. A Math. Theor. 2008, 41, 185208.

39. Cherniha, R.; Pliukhin, O. New conditional symmetries and exact solutions of reaction-diffusion-convection equations with exponential nonlinearities. J. Math. Anal. Appl. 2013, 403, $23-37$.

40. Cherniha, R. New Q-conditional symmetries and exact solutions of some reaction-diffusion-convection equations arising in mathematical biology. J. Math. Anal. Appl. 2007, 326, 783-799. 
41. Kunzinger, M.; Popovych, R.O. Is a nonclassical symmetry a symmetry. In Proceedings of the 4th Workshop "Group Analysis of Differential Equations and Integrability", Protaras, Cyprus, 26-30 October 2008.

42. Olver, P.J. Direct reduction and differential constraints. Proc. R. Soc. Lond. A 1994, 444, 509-523.

43. Olver, P.J.; Rosenau, P. The construction of special solutions to partial differential equations. Phys. Lett. A 1986, 114, 107-112.

44. Pucci, E.; Saccomandi, G. On the weak symmetry groups of partial differential equations. J. Math. Anal. Appl. 1992, 163, 588-598.

45. Boyko, V.M.; Kunzinger, M.; Popovych, R.O. Singular reduction modules of differential equations. J. Math. Phys. 2016, 57, 101503.

46. Kunzinger, M.; Popovych R.O. Singular reduction operators in two dimensions. J. Phys. A 2008, 41, 505201.

47. Fushchich, V.I.; Serov, N.I.; Repeta, V.K. Conditional symmetry, reduction and exact solutions of the nonlinear wave equation. Dokl. Akad. Nauk Ukr. SSR 1991, 161, 29-34. (In Russian)

(C) 2016 by the authors; licensee MDPI, Basel, Switzerland. This article is an open access article distributed under the terms and conditions of the Creative Commons Attribution (CC-BY) license (http://creativecommons.org/licenses/by/4.0/). 